\title{
La educación ambiental en la agenda 21 local de Sagunto (Valencia)
}

\author{
Verdugo Perona, José Javier \\ joverpe@alumni.uv.es \\ García Gómez, Javier \\ Garcia-Gomez@uv.es> \\ Universidad de Valencia
}

Resumen: La Agenda 21 surge en la Cumbre de la Tierra que tuvo lugar en Río de Janeiro en 1992 y es un plan de acción mundial con normas tendentes a la consecución de un desarrollo sostenible desde el punto de vista social, económico y ecológico. Plantea entre sus objetivos la participación ciudadana, pero para que ello sea posible, es necesaria la educación ambiental para generar en la población actitudes de respeto con el ambiente. Por ello es necesario establecer actuaciones que propicien la educación. La incorporación de las Agendas 21 locales ha sido muy heterogénea entre países y regiones y sus resultados no han sido suficientemente valorados. El presente trabajo forma parte de la investigación fin de Master realizada por el primer autor y dirigida por el segundo. En él se trata de analizar en qué medida en la ejecución de las Agendas locales 21 se incorpora la educación ambiental, para lo cual se investiga su realización en la población de Sagunto (Valencia-España).

Palabras clave: Educación Ambiental. Agenda 21 local.

Abstract: Agenda 21 comes at the 1992 Earth Summit in Rio de Janeiro and it is a global accion plan with rules aimed at achieving sustainable development from a social, economical and ecological point of view. Citizen participation is one of its objectives but, to make this possible, environmental education it is necessary to generate respect attitudes to environment in population. Therefore, it is needed to establish actions that propitiate education. The incorporation of Local Agenda 21 has been very heterogeneous across countries and regions and their results have not been sufficiently valued. This work is part of the Master's degree final research conducted by the first author and directed by the second. It tries to analyze to what extent is environmental education incorporated in the implementation of Local Agenda 21, for which purpose its realization in the town of Sagunto (Valencia-Spain) is investigated.

Key Words: Environmental Education, local Agenda 21.

(Fecha de recepción: junio, 2012, y de aceptación: septiembre, 2012)

DOI: 10.7203/DCES.26.1930 


\section{Introducción}

El origen de la Agenda 21 la podemos encontrar en la Cumbre de la Tierra. Después de la publicación del "Informe Brundtland", en 1989, la Organización de las Naciones Unidas comenzó a planificar la Conferencia sobre el Medio Ambiente y el Desarrollo. En ella se contó con la participación de numerosos expertos de todo el mundo entre los que se encontraban organizaciones no gubernamentales, grupos de empresarios, docentes, asociaciones femeninas, grupos indígenas y otros colectivos.

La conferencia se dio a conocer como Cumbre de la Tierra y tuvo lugar en Río de Janeiro del 3 al 14 de junio de 1992. Ésta fue un éxito sin precedentes que congregó al mayor número de jefes de Gobierno con el objetivo de establecer una alianza mundial nueva y equitativa mediante la creación de nuevos niveles de cooperación entre los Estados.

En esta Cumbre se aprobaron cinco documentos que constituyen el plan de acción de las Naciones Unidas en pro del desarrollo sostenible y son los siguientes: convención Marco de las Naciones Unidas sobre Cambio climático, convenio sobre Biodiversidad, Declaración de los bosques, Declaración sobre Medio Ambiente y Desarrollo.

En esta última se proclaman 27 principios en los que se definen los derechos y deberes de las naciones en la búsqueda del progreso y del bienestar de la humanidad y que darán lugar más adelante a la "Carta de la Tierra", una declaración de principios éticos fundamentales para la construcción de una sociedad global justa, sostenible y pacífica en el Siglo XXI.

De entre los 27 principios que se aprobaron en esta declaración se deben destacar, por su interés en el presente estudio, el principio 10 en el que se indica que "El mejor modo de tratar las cuestiones ambientales es con la participación de todos los ciudadanos interesados, en el nivel que corresponda. En el plano nacional, toda persona deberá tener acceso adecuado a la información sobre el medio ambiente de que dispongan las autoridades públicas, incluida la información sobre los materiales y las actividades que encierran peligro en sus comunidades, así como la oportunidad de participar en los procesos de adopción de decisiones. Los Estados deberán facilitar y fomentar la sensibilización y la participación de la población poniendo la información a disposición de todos. Deberá proporcionarse acceso efectivo a los procedimientos judiciales y administrativos, entre éstos el resarcimiento de daños y los recursos pertinentes."

El quinto documento es el Programa o Agenda 21, consistente en un plan de acción mundial con normas tendentes a la consecución de un desarrollo sostenible desde el punto de vista social, económico y ecológico. Se compone de un preámbulo y cuarenta capítulos en los que se abordan los problemas existentes en relación con el medio ambiente y el desarrollo, asignados a cuatro secciones diferentes, categorizadas de la siguiente manera: dimensiones sociales y económicas, conservación y gestión de los recursos para el desarrollo, fortale- 
cimiento del papel de los grupos principales y medios de ejecución.

De entre todos los capítulos que componen la Agenda 21, destacaremos dos por su especial relevancia con el tema que nos ocupa. El primero de ellos es el capítulo 28 (Iniciativas de las autoridades locales en apoyo del Programa 21), en el que se justifica la necesidad de la actuación local para la consecución de un desarrollo sostenible y que dio lugar a un programa concreto, la Agenda 21 Local:

"Como tantos de los problemas y de las soluciones de que se ocupa el Programa 21 se relacionan con las actividades locales, la participación y cooperación de las autoridades locales constituirán un factor determinante para el logro de los objetivos del Programa."

El otro capítulo de gran importancia es el número 36 (Fomento de la Educación, la Capacitación y la Toma de Conciencia), en el que, tomando los principios fundamentales de la Declaración y las recomendaciones de la Conferencia Intergubernamental de Tbilisi sobre Educación Ambiental (1977), se establecen las siguientes propuestas:

- Reorientación de la educación hacia el desarrollo sostenible.

- Aumento de la conciencia del público.

- Fomento de la capacitación.

Aunque, como ya se ha comentado, el origen de la Agenda 21 se encuentre en la Cumbre de las Naciones Unidas sobre el Medio ambiente y el Desarrollo (Cumbre de la Tierra. Río de Janeiro,
1992), otros muchos acontecimientos históricos en los que se han alcanzado diferentes acuerdos y tratados internacionales han influido significativamente, no sólo en su creación, sino también en la aplicación de nuevas mejoras que han propiciado su evolución para alcanzar los compromisos adquiridos de una forma más exitosa.

En la tabla 1, se muestran los eventos más importantes relacionados con la Agenda 21, así como los objetivos o acuerdos adoptados en cada una de ellos.

Desde la creación de la Unión Internacional para la Conservación de la Naturaleza (UICN), en 1948, se fueron extendiendo las ideas conservacionistas, pero no fue hasta los años 70 cuando se comienza a mostrar una mayor preocupación mundial por los problemas ambientales. Aunque ya se hablara con anterioridad, se acepta comúnmente que el inicio de la Educación Ambiental tuvo lugar a principios de década, concretamente en la Conferencia de Naciones Unidas sobre el Medio Humano (Estocolmo, 1972), el mismo año de la publicación del informe "Los límites del crecimiento". Dada la preocupación creciente por la conservación, se crea el Programa de Naciones Unidas para el Medio Ambiente (PNUMA) que conjuntamente con la UNESCO crea, en 1975, el Programa Internacional de Educación Ambiental (PIEA).

Desde entonces, se han venido celebrando congresos y conferencias internacionales sobre educación ambiental, como el Coloquio Internacional sobre Educación relativa al Medio Ambiente (Belgrado, 1975), la Conferencia 


\section{Tabla 1. Hitos históricos de la Agenda 21}

\begin{tabular}{|cl} 
AÑO & EVENTO \\
1972 & $\begin{array}{l}\text { Conferencia de } \\
\text { Estocolmo }\end{array}$
\end{tabular}

1973-76 I Programa

Comunitario de

Acción en Medio

Ambiente

\begin{tabular}{|ll}
$1977-81$ & $\begin{array}{l}\text { II P. Comunitario de } \\
\text { Acción en M.Ambiente }\end{array}$ \\
\hline $1982-86$ &
\end{tabular}

\begin{tabular}{|ll}
\hline $1982-86$ & $\begin{array}{l}\text { III P. Comunitario } \\
\text { de Acción en } \\
\text { Medio Ambiente }\end{array}$ \\
\hline 1984 & $\begin{array}{l}\text { Comis. Mundial sobre } \\
\text { M. Ambiente y Des. }\end{array}$ \\
\hline
\end{tabular}

1987-92 IV P. Comunitario de Acción en M. Ambiente

$1992 \quad \begin{aligned} & \text { Libro Verde Medio } \\ & \text { Ambiente Urbano }\end{aligned}$

\begin{tabular}{ll} 
1992-99 & $\begin{array}{l}\text { Conferenc. de Río: } \\
\text { "La Cumbre de la } \\
\text { Tierra" }\end{array}$ \\
\hline
\end{tabular}

1992-2000 V P. Comunitario de Acción en M. Ambiente de la UE "Hacia un Desarrollo Sostenible"

$1994 \quad \begin{aligned} & \text { I Conferencia de } \\ & \text { Ciudades Europeas } \\ & \text { Sostenibles. Aalborg } \\ & \text { (Dinamarca) }\end{aligned}$

$1996 \quad \begin{aligned} & \text { II Conf. de Ciudades } \\ & \text { uropeas Sostenibles. } \\ & \text { Lisboa (Portugal) }\end{aligned}$

$\begin{array}{ll}1997 & \begin{array}{l}\text { Cumbre Extraord. } \\ \text { Río+5. N.York. (EEUU) }\end{array}\end{array}$

\begin{tabular}{ll}
2000 & $\begin{array}{l}\text { III Conf. de Ciudades } \\
\text { Europeas Sostenibles. } \\
\text { Hannover. (Alemania) }\end{array}$ \\
\hline
\end{tabular}

2001-12 VI Prog. de Acción en Materia de M.Ambiente de la UE

2002 Conf. Mundial Río+10. II Cumbre de la Tierra. Johannesb. (Sudáfrica)

2004 IV Confer.de Ciudades y Pueblos Sostenibles. Aalborg +10

2010 VI Conf. de Ciudades y Pueblos Sostenibles.

Dunkerque. (Francia)

\section{OBJETIVOS MÁS IMPORTANTES}

Se crea el concepto problemática ambiental global al hablar de los problemas mundiales más importantes a los que se enfrenta la humanidad y se pone de manifiesto en el informe "Los Límites del Crecimiento" que de mantenerse las tendencias actuales de crecimiento el planeta colapsará en los próximos 100 años.

Se establecen los principios generales que deben regir la política comunitaria, entre los que destacan el de "prevenir el lugar de corregir" y el de "quien contamina paga". Se establecen tres líneas de acción: reducir y prevenir la contaminación, mejorar el entorno natural y desarrollar acciones en el seno de las organizaciones internacionales.

Se reafirman los objetivos del primer programa y se amplían algunos aspectos como la protección y gestión del espacio natural y la protección de la fauna y la flora.

Se amplían las líneas de actuación, como la integración del Medio ambiente en el resto de políticas comunitarias y la evaluación de los efectos ambientales de determinadas actividades productivas.

Se presenta el Informe Brudtland, titulado: "Nuestro futuro común", donde se consolida el concepto de Desarrollo sostenible, propugnando la armonía entre humanidad y naturaleza.

Se reafirma la necesidad de integrar la política ambiental en el resto de políticas comunitarias y pone de manifiesto el escaso grado de aplicación de las normas comunitarias.

Documento creado por la Comisión Europea para analizar la problemática ambiental urbana y las raíces de la misma.

Se plantea por primera vez la necesidad de alcanzar una política ambiental integrada para alcanzar un desarrollo sostenible, llegándose a un amplio acuerdo. Se elabora el Programa 0 Agenda 21 y se crea la Comisión para el Desarrollo Sostenible.

Integra las políticas ambientales adoptadas en la Cumbre de la Tierra en el resto de políticas económicas y sociales de la UE. Se amplía la gama de instrumentos disponibles para poner en práctica las políticas de protección ambiental y potenciar el grado de sensibilización e información de los ciudadanos en cuestiones de desarrollo sostenible.

Se crea la Carta de Aalborg partiendo de los principios establecidos en la Agenda 21. Pretende integrar los principios de sostenibilidad en todos los niveles, sobretodo en el ámbito local. Los firmantes de la Carta de Aalborg se comprometen a participar en un Plan de Acción a largo plazo hacia la Sostenibilidad (Agenda 21).

Se realiza el seguimiento de los acuerdos alcanzados en Aalborg y el grado de implantación de las Agenda 21 Local en los diferentes países europeos, promoviéndose el intercambio de experiencias entre municipios.

Revisión de los acuerdos alcanzados en la Cumbre de Río de 1992 y ratificando su programa de acción como el mejor para conseguir el desarrollo sostenible.

Se evalúan los proyectos existentes y los principios de sostenibilidad establecidos y se firma la Declaración de Hannover, en la que estableciéndose el liderazgo de la ciudad como motor de cambio a nivel local, reafirmándose el compromiso con la A. Local 21.

Inspirado en el programa anterior, el objetivo es proponer un Programa Marco General, jurídicamente vinculante, que se complete con un Plan de Acción Nacional en materia de medio ambiente en cada estado miembro.

En ella se analizan los avances logrados desde la Cumbre de la Tierra en 1992 y se aprueba un Plan de Acción que no fue del agrado de las ONG's por falta de metas y plazos para eliminar la contaminación, mejorar los servicios de agua potable y aumentar prod. de energía limpia.

Se revisan los 10 años de acción local en pro de la sostenibilidad y se establecen nuevos compromisos, siendo el primero de ellos el de impulsar procedimientos de toma de decisiones a través de una mayor democracia participativa.

Se adoptan dos declaraciones políticas: "Declaración de Dunkerque 2010 sobre Sostenibilidad Local", en la que se exige que se dé prioridad a una inversión y gestión de recursos más eficiente y sostenible; "Llamada a la acción Dunkerque 2010", donde se reconoce que para llegar a una economía de bajo carbono, resiliente y sostenible en Europa, es necesaria la integración de los gobiernos locales y regionales. 
Intergubernamental de Tbilisi (1977), el Congreso Mundial sobre Educación y Formación Ambiental (Moscú 1987), la Conferencia de las Naciones Unidas sobre Medio Ambiente y Desarrollo (Río de Janeiro, (1992), y la Conferencia Internacional Medio Ambiente y Sociedad: Educación y Sensibilización para la Sostenibilidad (Tesalónica, 1997).

Por lo que a España se refiere, la historia de la educación ambiental se divide en tres periodos (Ministerio de Medio Ambiente, 1999):

I. Inicios. Son inicios distintos en cada comunidad autónoma y las aportaciones desde la educación formal y no formal son muy diversas, lo que marcan el desarrollo de la educación ambiental en cada comunidad. Su expansión surge con la transición democrática y va unida a los movimientos ecologistas, conservacionistas y excursionistas y, en el ámbito escolar, al Movimientos de Renovación Pedagógica.

II. Fase de institucionalización. Durante los años 80 se generaliza la puesta en marcha de actividades, programas y equipamientos, públicos y privados, como granjas escuela, aulas de naturaleza o centros reinterpretación, ampliándose los destinatarios a grupos de adultos.

III. Etapa de replanteo crítico. Se inicia a partir de los años 90, con mayor conocimiento de la problemática ambiental que lleva a exigir criterios de calidad en los programas y actividades que se desarrollan, subrayando la nece- sidad de una educación ambiental que llegue a toda la sociedad y profundice en los principios de sostenibilidad.

Con la elaboración del "Libro Blanco de la Educación Ambiental en España" (1999) se pretende dar un nuevo impulso a la educación ambiental incidiendo, entre otros aspectos, en el fomento de la participación ciudadana en las iniciativas ambientales, como medio para alcanzar el consenso social que garantice el progreso hacia la sostenibilidad.

\section{Contexto regional y local}

\subsection{La Agenda 21 Local en Europa.}

En Europa es el ICLEI (The Internacional Council for Local Enviromental) el organismo encargado de promover la A21L mediante la creación de la Red Europea de Ciudades y Pueblos Sostenibles (Carta de Aalborg, 1994).

Desde su creación, la respuesta de los municipios europeos en cuanto a la implantación de la A21L ha sido todo un éxito, siendo el continente líder en su implantación. En el año 2002, 5.292 municipios de 36 países europeos ya se habían comprometido con la $\mathrm{A} 21 \mathrm{~L}$, lo que representaba más del $80 \%$ de los resultados mundiales. A ello ha contribuido la existencia de las campañas nacionales comentadas anteriormente, teniendo una implicación fundamental para la difusión de estos procesos, pues supone un apoyo de los gobiernos nacionales, incluyendo el apoyo financiero, para la implantación de este tipo de programas. Sólo los países con campañas nacionales suman 2.011 procesos 
de implantación de A21L, que es el 38\% de total de procesos que se desarrollan en Europa.

Las implantaciones entre los diferentes Estados no se llevan al mismo ritmo. Atendiendo a la rapidez de implantación de la A21L los países participantes pueden clasificarse en tres grupos:

- El primer grupo lo componen los países pioneros como Suecia, Reino Unido y Holanda, y el resto de países escandinavos, Dinamarca, Noruega y Finlandia, que comenzaron algo más tarde.

- En el segundo grupo se incluyen Alemania y Austria. Con una respuesta más tardía y débil.

- En el último grupo se encuentran los países mediterráneos (Italia, Portugal, España y Francia) e Irlanda, cuyo interés por la A21L se inicia a finales de los años 90 .

Estudios más recientes muestran que los países mediterráneos como España (Echebarría et al. 2004; Barrutia et al. 2007) e Italia (Sancassiani, 2005) que se iniciaron en la A21L más tarde, se han aprovechando de las experiencias previas del resto de participantes, facilitándoles el camino y permitiéndoles avanzar de una manera más rápida y sólida que los países pioneros, como por ejemplo, Suecia y Reino Unido, que han visto reducirse el número de municipios inmersos en A21L. Sin embargo, esta reducción no quiere decir que lo procesos de desarrollo sostenible se están abandonando en dichos países, sino que son sustituidos por otro tipo de propuestas (como los planes comunitarios o los planes estratégicos) cuyo enfoque se centra en un planteamiento más global y menos ambiental. Algo que no ocurre con los países mediterráneos que siguen utilizando la A21 como instrumento para repensar las políticas locales, para reformular programas y para intentar nuevas formas de participación comunitaria (Castiella y Subirats, 2007).

Por lo general, los países más avanzados en materia medioambiental (Suecia, Noruega, Finlandia y Dinamarca) son los que presentan un mayor grado de implantación y desarrollo de la A21L, con la excepción de Reino Unido que, pese a tener poca tradición en política ambiental, sí presenta una elevada implantación de la A21 debido a la exigencia de su implantación en la normativa legal. De este modo, las autoridades locales británicas utilizaron la A21L para intentar lograr una modernización democrática y para reafirmar y ampliar sus competencias, que habían sido drásticamente reducidas durante el Gobierno Tatcher (Jonas et al., 2004:152)

\subsection{La Agenda 21 Local en España.}

Aunque España se vinculó con el objetivo del Desarrollo Sostenible con la aprobación de la Constitución Española en 1978, (artículo 45), fue con su entrada en la Unión Europea el 1 de Enero de 1986 y la necesidad de adaptarse a los nuevos requerimientos, lo que verdaderamente propició una reforma normativa y un gran desarrollo en política ambiental. Aun así, España se encuentra a mucha distancia de lo que sería el perfil ambiental de la mayoría de los 
socios europeos, como Holanda, Suecia o Dinamarca, debido a la escasa cultura y tradición medioambiental. Esto se refleja en el tardío interés en España por la Agenda 21 Local, que no se dio hasta finales de los 90 , aunque posteriormente el número de municipios firmantes de la Carta de Aalborg tuvo un rápido crecimiento.

En el caso español, a diferencia de lo que ocurre en otros países europeos, no es el Estado el encargado de impulsar esta iniciativa. El modelo de Estado Autonómico español atribuye competencias ambientales a la administración central, a las autonómicas y a la administración local. Esto ha hecho que en la implantación de la A21L las responsabilidades queden repartidas y el grado de implantación sea muy desigual entre las diferentes administraciones españolas. La Estrategia Española de Desarrollo Sostenible, aprobada en el año 2002 como adaptación a la Estrategia Europea de Desarrollo Sostenible, es más un documento teórico que práctico en el que se expresa el deseo de que las autoridades locales se impliquen en la $\mathrm{A} 21 \mathrm{~L}$, sin ofrecer ningún tipo de ayudas. Así pues, la administración central no ha tenido un papel muy activo y han sido las Diputaciones Provinciales, las Mancomunidades y, sobre todo, los propios municipios los principales impulsores de los procesos de Agenda 21 Local.

En 1998, el 58\% de los grandes ayuntamientos españoles (población de más de 100.000 habitantes y/o capitales de provincia) ignoraban la existencia de la
A21L, el 24\% la conocían pero no habían adoptado medidas, mientras que solamente un $18 \%$ había iniciado el proceso de elaboración. Un año más tarde, el porcentaje de municipios que ignoraban qué era la A21L había descendido 17 puntos. El porcentaje de grandes ayuntamientos que habían adoptado medidas pasó del $18 \%$ al $31 \%$. (Tabla 2 )

\begin{tabular}{|c|c|c|}
\hline \multicolumn{3}{|c|}{$\begin{array}{l}\text { Tabla 2. Respuestas a A21L } \\
\text { (Grandes ayuntamientos) }\end{array}$} \\
\hline & 1998 & 1999 \\
\hline La ignoran & $58 \%$ & $41 \%$ \\
\hline $\begin{array}{l}\text { La conocen, pero no } \\
\text { adoptan medidas }\end{array}$ & $24 \%$ & $28 \%$ \\
\hline Adoptan medidas & $18 \%$ & $31 \%$ \\
\hline Total & $100 \%$ & $100 \%$ \\
\hline
\end{tabular}

En 1998 los municipios firmantes de la Carta de Aalborg eran aproximadamente sesenta, dos años después la cifra había alcanzado los ciento cincuenta municipios y en el año 2002 ya eran cuatrocientos doce, según los datos de la Federación Española de Municipios y Provincias. Los datos ofrecidos tras las IV Conferencia Europea de Ciudades Sostenibles (Aalborg+10) indican que en España han firmado o realizado una declaración de intenciones con dicha Carta un total de 355 Ayuntamientos, Regiones, Provincias, Mancomunidades o Diputaciones ${ }^{1}$, lo que hacen que sean

\footnotetext{
${ }^{1}$ http://www.aalborgplus10.dk/default.aspx?m=2\&i=308
} 
más de mil los municipios en las distintas fases de la A21L.

Este incremento en el número de municipios participantes en la A21L viene determinado, entre otros, por dos factores principales: la difusión y el intercambio de información sobre contenidos y experiencias en la elaboración de la A21L y la capacidad de liderazgo institucional.

El primero de los dos factores se produce a través de las instituciones que juegan un papel activo en la implantación de la Agenda 21 Local, promocionando sus experiencias en foros, redes intermunicipales o reuniones científicas, y mediante la interacción multilateral de redes de administraciones locales que operan en ámbito internacional, nacional y supramunicipal.

Las redes municipales, como la Xarxa de Ciutats i Pobles cap a la Sostenibilitat (Barcelona) o la Red Vasca de municipios hacia la sostenibilidad UDALSAREA 21, incluyen municipios que trabajan juntos por un desarrollo sostenible, favoreciendo el flujo de información y el intercambio de recursos a nivel intermunicipal. Con el objetivo de crear un foro de debate y que se diera este intercambio de experiencias entre las distintas redes que trabajaban en los proceso de A21L a nivel autonómico, regional y local en España se crea en 2005 la Red de Redes de Desarrollo Local Sostenible (RdR/DLS). Actualmente, esta red representa a más de 2.700 municipios, con una población de 24 millones de habitantes. Está formada por 18 redes más la Federación Española de Municipios y Provincias (FEMP), la Red Civitas España y Por- tugal y expertos, ostentando el Ministerio de Medio Ambiente, y Medio Rural y Marino el Secretariado. (Tabla 3)

En el estudio de la OSE (2011), se señala que durante el periodo 2000 2006 se constituyen la mayoría de las redes analizadas (14) y que entre los proyectos diseñados y ya desarrollados por estas redes destacan los de carácter puramente ambiental; en segundo lugar los socioeconómicos, y a mucha distancia de estos aquellos proyectos orientados a la mejora de la gobernanza. Igualmente se recoge que todas las redes organizan jornadas formativas sobre temas de interés en referencia al desarrollo sostenible local.

El segundo factor, determinante en el incremento Agenda 21 Locales, es el liderazgo institucional, ha tenido mejor acogida entre aquellos actores, ya sean municipales, supramunicipales o sociales, que han visto la oportunidad de utilizar la A21L para redefinir el modelo de desarrollo local y ganar visibilidad institucional. Ejemplos de este liderazgo institucional pueden verse en el papel desempeñado por las Diputaciones o los por propios Ayuntamientos.

Si el liderazgo lo ejercen las Diputaciones tiene como ventaja que, al implantar la A21L en un municipio, intervienen la administración local y la provincial, y esta última lo hace también en otros procesos por lo que hay mayor facilidad de intercambio de información. Por otro lado, al no ejercer control directo en el proceso de implantación, es necesaria una mayor voluntad política municipal para que éste llegue a buen término. Sin embargo, si son los ayuntamientos los que toman la inicia- 


\section{Tabla 3. Red de Redes de Desarrollo Local Sostenible (RdR/DLS)}

Red Provincial de Ciudades Sostenibles (Huelva).

Xarxa Balear de Sostenibilitat (Islas Baleares).

Programa de sostenibilidad ambiental Ciudad 21 (Andalucía).

Red de municipios sostenibles de la provincia de Jaén.

Xàrcia de municipis valencians cap a la sostenibilitat (Valencia).

Red de Agendas 21 locales cordobesas.

Red de entidades locales del Altoaragón por la sostenibilidad - Rete 21 (Diputación Provincial de Huesca).

CILMA - Consell d'Iniciatives Locals per al Medi Ambient de les comarques de Girona.

Red Vasca de municipios hacia la sostenibilidad - UDALSAREA 21.

Red Local de Sostenibilidad de Cantabria.

Red de Municipios sostenibles de la región de Murcia.

Red de Ciudades y Pueblos para la Sostenibilidad de la Provincia de Zaragoza.

Red Granadina de Municipios hacia la Sostenibilidad.

Alicante Natura Red Provincial de Agenda 21.

Red Astuaria21.

Red Civitas España y Portugal.

Federación Española de Municipios y Provincias (FEMP).

Ministerio de Medio Ambiente, y Medio Rural y Marino (Secretaría).

Fuente: Elaboración propia a partir de los datos publicados por RdR/DLR.

tiva de implantar una A21L se desprende un compromiso político muy elevado, que puede verse muy influenciado por los cambios en el gobierno local.

\subsection{La Agenda 21 Local en la Comuni- dad Valenciana.}

El compromiso de la Generalitat Valenciana con el desarrollo sostenible se hace patente en 1995 durante la II
Conferencia de Ministros y Responsables Políticos de las Regiones de la Unión Europea en materia de Medio Ambiente, celebrada en Valencia, y en la que se aprobó la Carta de Valencia, documento en el que se pone de manifiesto la responsabilidad de las Regiones de la Unión Europea en la consecución de un desarrollo sostenible. Sin embargo, no fue hasta el año 2002 cuando realmente 
la Generalitat estableció un marco para avanzar hacia un desarrollo más sostenible con la aprobación de la Estrategia para el Desarrollo Sostenible de la Comunidad Valenciana, adaptándose con ella a la Estrategia Española de Desarrollo Sostenible.

Con anterioridad, la Diputación de Valencia ya se había comprometido a potenciar el desarrollo sostenible en los municipios de su provincia tras suscribe en 1999 a la Carta de Aalborg, involucrándose de este modo en las iniciativas de A21L. Un año después, el 30 de mayo de 2000, se aprueba la Carta de Xàtiva, documento en el cual se establece el compromiso de los municipios firmantes de adherirse a la Carta de Aalborg y desarrollar planes de acción que garanticen un Desarrollo Sostenible y la necesidad de crear una Red de Municipios Valencianos hacia la Sostenibilidad, que integre a la Diputación y a los municipios valencianos, así como entidades públicas o privadas que decidan adherirse. Como fruto de la Carta de Xàtiva, el día 6 de marzo de 2001 se constituye la Xàrxia de Municipis Valencians cap a la Sostenibilitat como plataforma de intercambio de experiencias entre los municipios de la provincia de Valencia que tienen previsto implantar o ya están implantado la Agenda 21 Local.

Según los datos recogidos en el documento: "La Experiencia de la Xàrxia de Municipis Valencians cap a la Sostenibilitat (2001/2005)", en el año 2005 un total de 183 entidades locales, 5 mancomunidades y 34 empresas se habían adherido a la Carta de Xàtiva, aunque únicamente 67 de los municipios se encontraban desarrollando en la práctica la A21L, muchos de los cuales se encontraban en la fase inicial.

Por otro lado, la Diputación de Alicante comienza en el año 2003 a trabajar en una Red Provincial de Agendas 21 y dos años después, el día 3 de febrero de 2005, se aprueba por el Pleno de la Diputación la creación de Alicante Natura Red Provincial de Agenda 21. En el mismo pleno se suscriben además a la Carta y los Compromisos de Aalborg, comprometiéndose a participar cooperando con los municipios de su provincia en el proceso de Agenda 21, y se refrendó la Carta Provincial, conocida como la Carta de Catí. Esta Carta sirve de marco de actuación e integración de la Diputación y de los diferentes municipios alicantinos que implicados en la A21L y en ella se establece el inicio, el ámbito y los objetivos de la Red Provincial de Agenda 21.

En el año 2008, tres años después de su creación, esta red contaba con 74 de los 141 municipios alicantinos. A día de hoy, y como se puede observar en la Figura 4, el 64\% del total de municipios forman parte de la red de Alicante Natura Red Provincial de Agenda 21, lo que abarca el $98 \%$ de la población de la Provincia de Alicante.

Del mismo modo que en los casos anteriores, pero varios años más tarde, la Diputación de Castellón también impulsa una red que incluye a los municipios de la Provincia que apuestan por modelos sostenibles de desarrollo. Participa activamente en dos Agendas 21 comarcales, como son la Agenda 21 Espadán-Mijares, que se inicia en el año 2001, y la Agenda 21 Els Ports, 
que comienza a principios de 2007 , pero no es hasta finales del 2008 cuando empiezan a realizarse las acciones para definir el modelo de Red a implementar, así como sus características y funcionamiento. De este modo se crea, tras la adhesión a la Carta de Albocàsser el 20 de octubre de 2010, la Red de Municipios hacia la Sostenibilidad de la provincia de Castellón (REDCAS) como plataforma para el intercambio de conceptos, experiencias, instrumentos, metodologías y proyectos encaminados a conseguir el desarrollo sostenible de los municipios de la Provincia de Castellón.

Como puede apreciarse, en esta red no se incluyen únicamente los proyectos encaminados a implantar la A21L, sino que va más allá e incorpora a la Red iniciativas como la implantación de Sistemas de Gestión Ambiental (ISO 14.000 o EMAS), el desarrollo e implantación de medidas y/o auditorías tendentes a la implantación de prácticas de eficiencias energéticas, aprobación y aplicación de Planes o medidas de Movilidad en el municipio o Programas o Acciones de Educación Ambiental.

Aun así, en el Informe de Estado de Situación de las Iniciativas de Sostenibilidad en la Provincia de Castellón, llevado a cabo por la propia Red de Municipios hacia la Sostenibilidad de la Provincia de Castellón en el año 2009, se pone de relieve que se han desarrollado sobretodo procesos de Agenda 21 Local y acciones de Educación Ambiental. En total, 41 municipios expresan tener implantada la A21L en alguna de sus fases.

\subsection{La Agenda 21 Local en Sagunto.}

La Agenda 21 Local comienza en Sagunto en el año 1998 cuando, viendo las posibilidades de crecimiento del municipio de Sagunto, y con el objetivo de que este crecimiento se hiciera de una manera sostenible, se acuerda durante el pleno del 30 de abril adherirse a la Carta de Aalborg, mostrando con ello el compromiso del Ayuntamiento a participar en las iniciativas locales del Programa 21 y a desarrollar planes de acción a largo plazo que garanticen un desarrollo sostenible en su municipio.

Dos años después, el 27 de julio del 2000 , se acuerda durante el pleno ordinario de la corporación municipal la adhesión de Sagunto en la Red de Municipios Valencianos hacia la Sostenibilidad, lo que se denomina la Carta de Xàtiva, comprometiéndose a compartir sus experiencias en la implantación de la Agenda 21 Local con el restos de integrantes de la Red para la consecución de un desarrollo sostenible.

Durante los 3 años siguientes (20012003) se realizó la primera fase de la implantación de la A21L, el Diagnóstico o Auditoria ambiental, que incluyó:

- Identificación de los problemas socio-ambientales de Sagunto y sus causas.

- Interrelación de los diferentes aspectos sociales, económicos y ambientales que configuran una "radiografía" del municipio.

- Establecimiento de una serie de recomendaciones generales como respuesta a la problemática visualizada. 
Con la información económica, social y ambiental recogida durante la fase de Diagnóstico, parte de ella mediante encuesta ciudadana y analizada en el informe "Resultados del Cuestionario Sobre Medio Ambiente y Calidad de Vida del Municipio de Sagunto", se inició el trabajo con el Foro de la Agenda 21 para diseñar la segunda fase de la A21L, el Plan de Acción Local, que se desarrollo durante los años 2004-2005.

El Plan de Acción Local (esquema 2) está formado por 5 Líneas Estratégicas (Dinámica Institucional Sostenible, Gestión Integral del Medio Ambien- te, Revalorización de la Ciudad y Planificación Sostenible del Territorio, Establecimiento de Política integradora en materia sociocultural y Promoción de las Actividades Económicas y de Empleo) que incluyen los objetivos fundamentales en los que estructura el Plan de Acción. Estas Líneas Estratégicas, a su vez, se desarrollan en 20 Programas de Actuación, en los que se justifica los objetivos específicos de cada una de las líneas de actuación, que incluyen 165 proyectos o acciones concretas a realizar para alcanzar el objetivo de cada programa.

\title{
Esquema 2. Estructura Plan de Acción Local de Sagunto.
}

\section{Líneas estratégicas Objetivos fundamentales en los que se estructura el $\mathrm{Plan}$ de Acción Ambiental Municipal}

\section{0 programas de actuación \\ Justificación y objetivos específicos de cada una de las líneas de actuación}

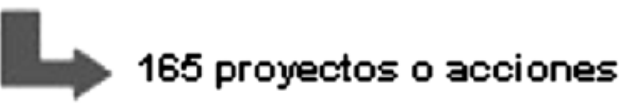

\begin{abstract}
Actuaciones o proyectos concretos a realizar para alcanzar el objetivo del programa
\end{abstract}

Fuente: Agenda 21 Local de Sagunto ${ }^{2}$

$\mathrm{Al}$ mismo tiempo que se iba realizando el Plan de Acción Local, se comenzó a diseñar el Plan de Seguimiento de los procesos de $\mathrm{A} 21 \mathrm{~L}$, para lo que se crearon una serie de indicadores de sostenibilidad, asociados a cada una de las líneas estratégicas (tabla 4).
A principios del 2006, se realizó el "Primer Informe de Seguimiento del Plan de Acción Local", constituyendo la puesta en marcha de esta nueva fase, en la que ciudadanía y Ayuntamiento han de colaborar estrechamente. Para ello, se creó la Comisión de Seguimien-

\footnotetext{
${ }^{2}$ http://www.a21sagunt.org/index.php?option=com_content\&task=view\&id=42\&Itemid=84
} 


\section{Tabla 4. Relación de Indicadores de Sostenibilidad de cada línea estratégica}

\section{LÍNEA}

\section{ESTRATÉGICA}

Dinámica institucional

Sostenible

\section{INDICADORES}

Proporción de acciones contenidas en el PAL en ejecución y/o ejecutadas

Proporción del gasto municipal en 2006

Gasto del Ayuntamiento en Educación Ambiental

Satisfacción de la ciudadanía acerca de la calidad de vida

\section{Gestión Integral}

del Medioambiente
Consumo total de agua por habitante y día

Rendimiento de la red de distribución de agua potable

Calidad del Agua de Consumo

Consumo doméstico de agua por habitante y día

Consumo de agua en la industria

Consumo de agua en la agricultura

Porcentaje de aguas residuales depuradas que se reutiliza

Generación de residuos sólidos urbanos per capita

Proporción de residuos sólidos urbanos recogidos selectivamente

$\mathrm{N}^{\circ}$ de días con una calidad del aire mejorable o deficiente

$\mathrm{N}^{\circ}$ de veces en que se supera el umbral de inform. a la pobl. para el ozono

Nivel acústico de las calles con más tránsito

Consumo total de energía por habitante y año

Consumo doméstico de electricidad

Incorporación de energías renovables

$\mathrm{N}^{\circ}$ de empresas que disponen de un sist. de gestión ambiental

Ciudad y planificación Ocupación urbana del suelo

del territorio

Superficie de zonas verdes urbanas per capita

Superficie natural protegida

Proporción de calles con prioridad para viandantes y bicicletas

Proporción de personas que utilizan el transporte público

Tasa de motorización de la población

Política integradora en materia sociocultural
Porcentaje de estudiantes graduados en ESO que continúan su formación

Funcionamiento de los órganos de participación

Partidas presupuestarias destinadas a recursos sociales

Número de actividades culturales realizadas en el municipio
Actividades económicas y empleo
Nivel económico del municipio

Tasa de desempleo

Proporción de mujeres desempleadas

Superficie de comercios minoristas por habitante

Dependencia del urbanismo en la economía local

Fuente: Sistema de Indicadores de Sostenibilidad de Sagunto (2006) 
to, órgano compuesto por ciudadanos y ciudadanas, a través de los representantes del Foro, y el Ayuntamiento, a través de sus técnicos y políticos.

En dicho informe se comparaban las acciones del Plan de Acción Local, valorándose únicamente aquellas que por su bajo coste económico y fácil ejecución pudieran haber sido incluidas en el actual ejercicio, y la realidad municipal. En total se valoraron 98 acciones de las 165 posibles, una amplia mayoría de las que contiene el documento.

El informe concluye que el Ayuntamiento debe considerar el Plan de
Acción Local de manera más profunda y rigurosa, indicando que todavía están el la fase inicial de implementación del Plan de Acción Local. Algo más tarde, ese mismo año, se realizó un segundo Informe más meticuloso en el que se revisaron de nuevo el estado de ejecución de las acciones contenidas en el Plan de Acción, tomando como referencia el primer informe de seguimiento. El estado en que se encontraban en ese momento las diferentes acciones se resume en la tabla 5 y desde el año 2008 no se ha realizado ningún tipo de actuación en ninguna de las Líneas Estratégicas.

\section{Tabla 5. Estado de ejecución de las Acciones consideradas.}

\begin{tabular}{|l|c|c|}
\cline { 2 - 3 } & \multicolumn{2}{|c|}{$\mathbf{N}^{\mathbf{0}}$ ACCIONES } \\
\cline { 2 - 3 } & 1 $^{\text {er }}$ Informe seguimiento PAL & $2^{\circ}$ Informe seguimiento PAL \\
\hline No valorado & 2 & 9 \\
\hline No ejecutado & 30 & 66 \\
\hline Pendiente de ejecución & 7 & 12 \\
\hline En ejecución & 53 & 39 \\
\hline Ejecutado & 6 & 7 \\
\hline
\end{tabular}

Fuente: $1^{\mathrm{er}}$ y $2^{\circ}$ Informe de Seguimiento del Plan de Acción Local (2006).

\section{La Educación Ambiental en la Agenda 21 Local de Sagunto.}

La Agenda 21 Local es la herramienta más ampliamente utilizada para alcanzar el desarrollo sostenible a nivel local. Está basada principalmente en la participación ciudadana y por ello es de gran importancia sensibilizar a los ciudadanos y ciudadanas de la importancia de su implicación y proporcionarles los conocimientos y las capacidades necesarias para hacer frente a los problemas ambientales que nos afectan. De todo esto se encarga la Educación Ambiental, que se puede definir como:

“...un proceso permanente en el cual los individuos y las comunidades adquieren conciencia de su medio y aprenden los conocimientos, los valores, las destrezas, la experiencia y también la determi- 
nación que les capacite para actuar, individual y colectivamente, en la resolución de los problemas ambientales presentes y futuros". (Congreso Internacional de Educación y Formación sobre Medio Ambiente. Moscú, 1987).

En la Agenda 21 Local de Sagunto, se pueden ver concretadas las acciones de Educación Ambiental incluidas en el Plan de Acción Local a través de los indicadores de sostenibilidad local (tabla 5). Según se desprende de los datos que aparecen en la segunda revisión del Sistema de Indicadores de Sostenibilidad de Sagunto (2006), dos de estos indicadores de sostenibilidad presentan una estrecha relación con la educación ambiental: el gasto del Ayuntamiento en Educación Ambiental y el consumo doméstico de agua por habitante y día.

- Gasto del Ayuntamiento en Educación Ambiental.

Con este indicador se muestra el interés y confianza en el instrumento de la Educación Ambiental como medida eficaz de cambio y mejora continua de los hábitos y comportamientos sociales (individuales y colectivos) y está ligado con el Plan de Acción Local a través de diferentes acciones que pertenecen a tres de las cinco líneas estratégicas establecidas (tabla 6).

\section{Tabla 6. Acciones presentes en el Plan de Acción Local relacionadas con el gasto del Ayuntamiento en Educación Ambiental}

\begin{tabular}{|cl|}
\hline ACCIÓN & DESCRIPCIÓN \\
\hline 1.3.5. & Promoción del voluntariado \\
\hline 2.1 .11$. & Elaboración de una guía de buenas prácticas en la utilización del recurso del agua. \\
\hline 2.1 .13$. & Campaña de Educación Ambiental: uso sostenible del agua. \\
\hline 2.2 .5$. & Fomento de la minimización en origen \\
\hline 2.2 .7$. & Campaña de educación dirigida a la minimización en origen (residuos sólidos). \\
\hline 2.3 .7$. & Campaña de sensibilización sobre emisiones del tráfico rodado \\
\hline 2.4 .2$. & Promoción de energías renovables: creación de una oficina, campaña de información \\
\hline 2.6 .1$. & sobre ahorro energético y energías renovables. \\
\hline 2.6 .2$. & Fromoción de la implantación de los sistemas de gestión ambiental en las empresas. \\
\hline 3.1 .5$. & Difundir y promover criterios de arquitectura sostenible \\
\hline 3.2 .1$. & Campañas divulgativas sobre espacios naturales \\
\hline 3.2 .4$. & Colaboración con el Centro de Educación Ambiental de la Comunidad Valenciana \\
\hline 3.2 .5$. & Creación de un aula de la naturaleza en la Marjal Norte \\
\hline 3.4 .6$. & Campañas de educación vial y mayor cobertura sancionadora \\
\hline 4.2 .1$. & Programa de educación ambiental municipal \\
\hline 4.2 .4$. & Fomento de los programas de educación para la sostenibilidad \\
\hline 4.2 .5$. & Campaña de educación en valores \\
\hline
\end{tabular}

Fuente: Sistema de indicadores de sostenibilidad de Sagunto. Segunda revisión (2006) 
Hasta el año 2006, el presupuesto destinado a Educación Ambiental había aumentado considerablemente, pasando de 8.773,01 € en el año 2004 a un total de 21.995,21 en el año 2006 (tabla 7).

\begin{tabular}{|lccc|}
\hline \multicolumn{4}{|c|}{ Tabla 7. Gasto del Ayuntamiento en Educación ambiental } \\
\hline CONCEPTO & $\mathbf{2 0 0 4}$ & $\mathbf{2 0 0 5}$ & $\mathbf{2 0 0 6}$ \\
\hline Voluntariado & 7.408 & 6.851 & 19.995 \\
\hline Día de la bici & 313 & 1.300 & (sin información) \\
\hline Día del Árbol & 1.052 & 903 & 2.000 \\
\hline TOTAL & $8.773,01$ & $9.053,91$ & $21.995,21$ \\
\hline
\end{tabular}

Fuente: Sistema de indicadores de sostenibilidad de Sagunto. Segunda revisión (2006)

De todas las acciones vinculadas con este indicador hay cuatro, que por su carácter educativo en materia ambiental, deben ser destacas. Son las siguientes:

- Acción 3.2.4. "Colaboración con el Centro de Educación Ambiental de la Comunidad Valenciana (Marjal dels Moros)". Esta colaboración se centra en el programa de voluntariado Majal Viva y en visitas con los participantes en la Escola d'Estiu y se desarrolla en los meses de verano.

En la segunda revisión del Plan de Acción Local se consideró una actuación correcta, pero se propuso establecer un convenio estable durante todo el año, se solicitó un medio de transporte público que permitiera el acceso al Centro y que éste sea promocionado.

- Acción 3.2.5. "Creación de un aula de la naturaleza en la Marjal Norte. Se trata de un proyecto "Proyecto de adecuación ambiental y uso educativo de la Marjal de Almenara y zona húmeda de la desembocadura del río Mijares. En él se establece la creación de un aula de interpretación y educación ambiental en la Casa Penya, pera todavía no se encuentra abierto al público.

- Acción 4.2.1. Programa de Educación Ambiental Municipal. Se realiza mediante un convenio de colaboración entre el Ayuntamiento de Sagunto y Acció Ecologísta Agró. Este programa se inició en el año 2006 y ha sido renovado recientemente hasta el año 2015 . Cuenta con un presupuesto de $30.000 € /$ año, incluye:

- El Día del Árbol. Actividad ofertada a las escuelas del municipio, en la que niños y niñas de $5^{\circ}$ de primaria plantan aromáticas.

- El grupo de voluntariado ambiental la Colla Verda, que realiza actividades en los espacios naturales del municipio.

- El proyecto educativo Participa! Taller de participación que profundiza en los conocimientos sobre la Marjal dels Moros y que va dirigido a los IES de Sagunto. 
- Acción 4.2.4. "Fomento de los programas de educación para la sostenibilidad". El Departamento de Medio Ambiente organizó un Ciclo de Monográficos sobre Sostenibilidad y Medio Ambiente dirigido a toda la ciudadanía y que contó con la presencia de expertos de relevancia. Aunque se consideró una buena iniciativa, el Foro consideró que no se cumplían los objetivos de la acción y que se debían promocionar con mayor ahínco la formación y educación en materia de sostenibilidad.

El resto de Acciones dirigidas a la ciudadanía pretender principalmente concienciar y sensibilizar a la población a través de guías y trípticos, además de en los diferentes programas de voluntariado o en las Escolas d'Estiu que se realizan.

También hay que resaltar aquellas acciones que van dirigidas a la empresa, como la Acción 2.6.1 Promoción de la implantación de los sistemas de gestión ambiental en las empresas y la Acción 2.6.2. Fomento buenas prácticas ambientales en las empresas. Ambas acciones incluyen la creación de los Premios Cerverola de Medio Ambiente, dirigidos a la gestión empresarial, siendo una de sus líneas la "Gestión empresarial favorable al desarrollo sostenible". Por otro lado, la introducción de buenas prácticas ambientales en las empresas se realiza también a partir de los condicionantes mínimos que debe cumplir la empresa para la obtención de la licencia de actividades.

Desde el Foro, se consideró correcta la actuación pero se cree que la colaboración debería extenderse al resto del año, mediante la firma de un convenio estable. Por otro lado, se recordó que la acción incluía la colaboración del Ayuntamiento con el Centro previendo un medio de transporte público que permita el acceso al Centro, promocionándolo en las Oficinas de Turismo, etc.

- Consumo doméstico de agua por habitante y día

En este indicador se refleja el hábito de los ciudadanos de Sagunto en cuanto a ahorro personal de agua y su sensibilización ante los problemas ambientales locales. Se relaciona con el programa 2.1 de Optimización de la Gestión Integral del Agua, especialmente con las acciones mostradas en la tabla 8 .

\section{Tabla 8. Acciones presentes en el Plan de Acción Local relacionadas con el consumo doméstico de agua por habitante y día}

\section{ACCIÓN DESCRIPCIÓN}

2.1.1. Estudio de los recursos hídricos disponibles. Plan de gestión de los recursos hídricos.

2.1.4. Plan Municipal de Ahorro de Agua (ordenanza municipal de ahorro de agua).

2.1.11. Elaboración de una guía de buenas prácticas en la utilización del recurso del agua.

2.1.12. Control del consumo del agua.

2.1.13. Campaña de Educación Ambiental: uso sostenible del agua.

Fuente: Sistema de indicadores de sostenibilidad de Sagunto. Segunda revisión (2006) 
Dos de las acciones (2.1.11 y 2.1.13) relacionadas con el consumo doméstico de agua por habitante y día se encuentran relacionadas también con el Gasto en Educación Ambiental. En ambos casos, son actuaciones de sensibilización sobre el uso del agua.

De las tres acciones restantes destaca la siguiente:

- Acción 2.1.12. Control del consumo de agua. El Departamento de Mantenimiento es el encargado de llevar un control anual del consumo de agua y de proporcionar la información a la ciudadanía a través del sistema de indicadores. Este sistema incluye varios indicadores referidos al consumo de agua. En concreto los indicadores 5 a 11 :

1. Consumo total de agua por habitante y día.

2. Rendimiento de la red de distribución de agua potable.

3. Calidad del Agua de Consumo.

4. Consumo doméstico de agua por habitante y día.

5. Consumo de agua en la industria

6. Consumo de agua en la agricultura.

7. Porcentaje de aguas residuales depuradas que se reutiliza

Según los últimos datos disponibles (segunda revisión sistema de indicadores, 2006), los indicadores 5 y 10 siguen la tendencia deseada, disminuyendo el consumo de agua. Por otro lado, el indicador 6, que valora el agua que se pierde durante el reparto de aguas debido a fugas, averías y acometidas no controladas, sigue una tendencia contraria a la deseada, lo que indica que no se ha aumentado el rendimiento de la red de distribución de agua potable.

\section{Conclusiones}

La Agenda 21 Local es la herramienta más extendida para alcanzar el desarrollo sostenible. Se fundamenta en la participación ciudadana, para lo cual requiere que la población esté dispuesta a involucrarse y preparada para enfrentarse a los retos que se les presente durante su implantación.

Esta labor corresponde en parte a la Educación Ambiental. Es necesario que se realicen acciones destinadas a toda la población, cualquiera que sea su edad, en las que resalten la importancia de la implicación ciudadana y se establezcan los fundamentos sobre sostenibilidad, proporcionando los conocimientos, las actitudes y las aptitudes necesarias para participar.

Entre las acciones presentes en el Plan de Acción Local de la A21L de Sagunto destinadas a tal fin, destacan aquellas que abordan cuestiones sobre la gestión de los recursos y los residuos y los programas de voluntariado. Estas acciones, dirigidas a toda la población, aunque beneficiosas para concienciar, sensibilizar sobre la problemática local y favorecer la implicación ciudadana, no muestran el carácter interdisciplinar que conlleva el concepto de sostenibilidad, necesario para comprender la complejidad del medio ambiente.

La formación ciudadana en materia ambiental debería establecerse, principalmente, en las acciones 4.2.1 (Programa de Educación Ambiental Municipal) y 4.2.4 (Fomento de los Programas de Educación para la Sostenibilidad). En ellas deberían establecer los fundamentos sobre sostenibilidad y medio ambien- 
te que capacitaran a la población, cualquiera que sea su edad, para involucrarse en la implantación de la A21L. Sin embargo, el Programa de Educación Ambiental Municipal, que no es gestionado por el propio Ayuntamiento, sino por la asociación Acció Ecologista Agró, únicamente cuenta con una acción dirigida a toda la ciudadanía, que consiste en un programa de voluntariado, y dos actividades (el taller Participa! y el Día del Árbol) enfocadas exclusivamente para alumnos de secundaria y de $5^{\circ}$ de primaria respectivamente. Se realizan en colaboración con los centros educativos del municipio, lo que facilita la conexión con la educación formal, pero sólo van dirigidas a determinados cursos. Esto hace que se eche en falta otro tipo de actuaciones que integren a todos los cursos e impliquen a toda la comunidad educativa en el desarrollo local, como podría ser la implantación de Agendas 21 Escolares.

Por otro lado, las acciones dirigidas a fomentar la responsabilidad ambiental en la empresa son insuficientes, tal y como se indicó en el segundo informe de revisión del Plan de Acción Local. Esto hace necesaria la creación de nuevas iniciativas que, del mismo modo que la acción 3.1.5 (Difundir y promover criterios de arquitectura sostenible), se dirijan no sólo a empresarios, sino también a grupos profesionales que pudieran tener especial relación con los problemas ambientales, como pueden ser los agricultores y comerciantes del municipio, con el fin de promover actitudes responsables en el marco de su actividad.

Es destacable también el escaso aprovechamiento que se realiza de la coope- ración existente con el Centro de Educación Ambiental de la Comunidad Valenciana (acción 3.2.4) y que consiste en un voluntariado ambiental en la Majal dels Moros. Si bien es un buen modo de dar a conocer la riqueza ambiental del municipio e implicar a los habitantes del municipio en su conservación, se considera una acción insuficiente, sobretodo teniendo en cuenta que dicho centro está situado en el término municipal de Sagunto y en él se realizan multitud de acciones formativas en materia de Educación Ambiental.

En resumen, podría decirse que las acciones de Ecuación Ambiental presentes en el Plan de Acción Local muestran un carácter informativo y de sensibilización que contribuye a fomentar hábitos respetuosos con el medio ambiente, pero es necesario educar no sólo sobre los problemas derivados del consumo irracional de los recursos naturales y la contaminación, sino también mostrar la relaciones existentes con otro tipo de actividades políticas, económicas, sociales, culturales y ambientales. Todo ello con el fin de que se comprenda el verdadero significado del concepto de sostenibilidad y se conciencie de la necesidad de la implicación ciudadana, en todos los niveles, para alcanzar ese modelo de desarrollo.

Por último, la forma de evaluar el efecto de estas acciones en la ciudadanía es a través del consumo de agua por habitante y día, uno de los indicadores del sistema de indicadores de sostenibilidad. A él se le asigna un significativo carácter de educación ambiental/comunicación ciudadana y, según se describe en el sistema de indicadores de sosteni- 
bilidad, refleja los hábitos de los ciudadanos y ciudadanas en cuanto al ahorro personal de agua y sensibilización ante los problemas ambientales.

No obstante, para conocer con mayor precisión dicha sensibilización ambiental se deberían tener en cuenta otros indicadores (como el consumo de agua en la industria/agricultura o la proporción de residuos sólidos urbanos recogidos selectivamente) que aportaran información sobre los hábitos y costumbres, no sólo de la ciudadanía, sino de todos sectores de la población. El análisis de los datos proporcionados por estos indicadores serviría también para evaluar el resultado de las acciones de educación ambiental realizadas y ayudarían a establecer estrategias de educación ambiental adecuadas para cada caso.

Aunque este estudio se ha realizado exclusivamente en el municipio de Sagunto y sería necesario disponer de más datos para generalizar, podría servir de ejemplo de las acciones de Educación Ambiental que se desarrollan en las Agenda 21 Locales, teniendo en cuenta que este fue uno de los municipios pioneros, junto con Xàtiva, Bolbaite y Estivella, en incorporar estos procesos.

\section{Bibliografía}

AGUADO, I. y ECHEBARRÍA, M. (2003). La Agenda Local 21 como instrumento de sostenibilidad: la experiencia española. Revista española de estudios agrosociales y pesqueros, 199, 61-91.

ALICANTE NATURA. Red Provincial Agenda 21. Agenda 21, Red provin- cial de alicante. Diputación de Alicante.

ALICANTE NATURA. Red Provincial Agenda 21. 12 Pasos para la red de agenda 2l. Diputación de Alicante. AYUNTAMIENTO DE SAGUNTO. Auditoría ambiental de Sagunto. Diagnosis. Documento de síntesis. Diputación de Valencia.

AYUNTAMIENTO DE SAGUNTO.

Propuesta de normas reguladoras del Foro de la Agenda 21 Local de Sagunto. Diputación de Valencia.

AYUNTAMIENTO DE SAGUNTO.

Resultados del cuestionario sobre medio ambiente y calidad de vida del municipio de Sagunto.

AYUNTAMIENTO DE SAGUNTO (2005). Plan de Acción Local

AYUNTAMIENTO DE SAGUNTO (2006). Borrador segundo informe de seguimiento del Plan de Acción Local.

AYUNTAMIENTO DE SAGUNTO (2006). Primer informe de seguimiento del Plan de Acción Local.

AYUNTAMIENTO DE SAGUNTO (2006). Sistema de indicadores de sostenibilidad de Sagunto (segunda revisión).

BARRUTIA, J. M. y AGUADO, I. (2007). La Agenda 21 Local en Europa: una visión general. Ekonomíaz, $\mathrm{N}^{\circ} 64$, 72-91.

CASTIELLA, T. y SUBIRATS, J. (2007). De la agenda a la acción. El caso de Barcelona. Ekonomiaz: Revista vasca de economía, 64, 35-46.

ECHABARRÍA, M., BARRUTIA, J.M. y AGUADO, I. (2004). Local Agenda 
21: Progress in Spain. European Urban and Regional Studies, vol. 11, $n^{\circ} 3$, pp. 273-281. En Echebarría, M. FONT, N. y SUBIRATS, J. (2000). Local y sostenible. La Agenda 21 Local en España. Editorial Icaria. Barcelona. ICLEI (2002). Second Local Agenda

21 Survey. Iternational Council for Local Environemental Initiatives (ICLEI).

JONAS, A., WHILE, A. y GIBAS, D. (2004): State modernisation and local strategic selectivity alter Local Agenda 21: evidence from three northern English localities. Policy \& Politics, vol. 32, $\mathrm{n}^{\circ}$ 2, pp. 151-168. En Echebarría, M., Barrutia, J. M. y Aguado, I. (2007). La Agenda 21 Local en Europa: una visión general. Ekonomíaz, No 64, 72-91.

MINISTERIO DE MEDIO AMBIENTE (1999). Libro Blanco de la Educación Ambiental en España. Madrid. MMA.

OSE (2011). Informe de sostenibilidad en España 2011. Observatorio de la Sostenibilidad en España (OSE). SANCASSIANI, W. (2005). Local Agenda 21 in Italy: An effective governance tool for facilitating local communities' participation and promoting capacity building for sustainability. Local Environment, vol. $10, n^{\circ} 2$, april, pp. 189-200. En Echebarría, M., Barrutia, J. M. y Aguado, I. (2007). La Agenda 21 Local en Europa: una visión general. Ekonomíaz, No 64, 72-91.

UNESCO (1977). Conferencia Intergubernamental sobre Educación Ambiental. Tbilisi. (URSS). Octubre. Informe fianl. Doc. ED-MD. 49. París. UNESCO.

\section{Webgrafia}

Agenda 21 de Sagunto.

Alicante Natura. Red Provincial Agenda 21

Ayuntamiento de Sagunto.

Foro Agenda 21 Sagunto.

Ministerio de Agricultura, Alimentación y Medio Ambiente. Desarrollo de Medio Ambiente Urbano.

Observatorio de la Sostenibilidad en España (OSE).

ONU Programa 21.

Red de Municipios hacia la Sostenibilidad de la Provincia de Castellón. UNESCO.

Xàrcia de Minicipis Valencias Cap a la Sostenibilitat. 
